\title{
SOA Governance from an Enterprise Architecture Viewpoint
}

\author{
Thaíssa Diirr ${ }^{1}$, Leonardo Guerreiro Azevedo ${ }^{1,2}$, Flávia Maria Santoro ${ }^{1}$ \\ ${ }^{1}$ Post-Graduation Program in Informatics \\ Federal University of the State of Rio de Janeiro (UNIRIO), Brazil \\ ${ }^{2}$ IBM Research - Brazil \\ \{thaissa.medeiros, azevedo, flavia.santoro\}@uniriotec.br; lga@br.ibm.com
}

\begin{abstract}
To obtain business benefits resulting from the implementation of an SOA approach is not sufficient managing technical features. A strategy aligned to business should be considered as a basis for activities of implementation, validation, development and services management. A business case, a reference model and an architecture of the organization should be established. This paper proposes SOA governance processes. The processes were evaluated by SOA experts who argue that they would adopt them for SOA governance in their organizations.
\end{abstract}

Resumo. Para obter os beneficios corporativos com a implantação de uma abordagem SOA não é suficiente tratar características técnicas. Uma estratégia alinhada ao negócio deve ser considerada como base para as atividades de implementação, validação, desenvolvimento e gestão de serviços. Um caso de negócio, um modelo de referência e uma arquitetura da organização devem ser estabelecidos. Este trabalho propõe processos para governança SOA. Os processos foram avaliados por especialistas na área de SOA que argumentam que adotariam os mesmos para a governança SOA em suas organizações.

\section{Introduction}

According to Deler and Weinreich (2006), many studies in SOA focus on the creation and use of technologies for Web Services development. However, the adoption of such specifications (e.g., WSDL (http://www.w3.org/TR/wsdl), UDDI (http://www.uddi.org/, 2008), WS-* (Erl, 2005), SOAP (http://www.w3.org/TR/soap/) and related activities (e.g., creation, validation, implementation and management) by themselves do not suffice to obtain the expected benefits of a SOA initiative from a business perspective. They are not enough to ensure that the corporation's dynamism is consistent according to SOA principles. In other words, this view is more technical and has low adherence to business goals. The definition of a SOA strategy helps to focus SOA efforts, to clarify its expected results and to identify appropriate uses for services so as to foster business benefits. It is a good practice to establish a business case, define a reference model and build/update the enterprise architecture models. All previous tasks are addressed by applying a SOA governance approach. However, the deployment of an adequate SOA governance approach still faces several challenges, such as how to encapsulate business activities into services, how to manage service changes, the establishment of new responsibilities and architecture roles, how to define and use metrics to measure achieved results, and how to establish and implement policies and standards (Schepers et al., 2008; Kajko-Mattsson et al., 2007). 
SOA Governance is the definition, implementation and subsequent enforcement of a decision model and a structure of responsibilities which ensure that an organization pursues a SOA strategy and that all SOA initiatives walk together to meet the organization requirements (Marks, 2008). SOA strategy must be aligned to business objectives; SOA governance has to ensure the implementation of this strategy in accordance with principles and policies, through committees or work groups, governance processes, checkpoints, reviews and tools and technologies. SOA governance includes the deployment of a SOA initiative according to business processes, technology standards and business priorities. Finally, SOA governance explicitly involves stakeholders from business and IT in the decision-making process related to SOA (Marks, 2008). On the other hand, Enterprise Architecture is defined as the organizing logic for business processes, data, and technology (Ross, 2011). This leads to the necessity of defining a SOA governance approach that is totally aligned to the Enterprise Architecture.

This work proposes SOA governance processes from the perspective of Enterprise Architecture since it extends approaches in this context described in Section 2. The proposal was evaluated by SOA experts from different organizations. The results indicate ease of understanding of processes and their usefulness to organizations.

The remaining of this paper is structured as follows. Section 2 presents related work. Section 3 details the proposed processes for SOA governance. Section 4 describes the evaluation of the proposal. Section 5 presents the conclusions and future work.

\section{Related Work}

Niemann et al. (2010) propose a generic SOA governance model including: SOA goals, SOA as enterprise architecture and governance control cycle. SOA goals are derived from global IT goals and correspond to specialized business goals. These goals are: SOA compliance (adherence to internal, technical and legal regulations); alignment between business and IT (integration and adoption of IT processes in the business environment is crucial to the success of SOA), and longterm reliable operation (resulting from the management of SOA). SOA as enterprise architecture consists of processes such as production, operation and maintenance of services, beyond the technical view including registers and enterprise service bus. The SOA governance control cycle is the central part that implements and operates effective governance. The cycle represents crucial processes, including and involving organizational entities (roles and responsibilities and governance processes), governance policies, catalog of best practices, compliance observation and enforcement techniques, and a component for measuring SOA maturity. Niemann et al. (2010) also investigated and compared approaches to SOA governance proposed by academia and industry. The following concepts of governance that were considered in these proposals were identified: impact on the organization, SOA maturity model, new roles and responsibilities, best practices, metrics model, impact on people's behavior, SOA life cycle. SOA roadmap, policies catalog, services lifecycle, governance processes, policy enforcement mechanisms. The concepts presented by Niemanm et al. (2010) are generic and difficult to follow in practice. However, it addresses key aspects of SOA governance. Among these concepts, our proposal does not cover the SOA maturity model and best practices. On the other hand, we discuss the concepts relevant to the application of SOA governance, and the level of detail allows its practical application.

Schepers et al. (2008) define a SOA governance life cycle composed by the following processes: define SOA strategy; align the organization; manage services portfolio; manage services life cycle; manage SOA policies and manage service levels. The authors also point out 
some important issues of SOA: tracking of IT systems and services to ensure compliance with standards and legislation; creation of budget according to the property and costs of services; analysis of impacts on services maintenance related to service consumers; quality assurance in services design and implementation; change of team behavior for the adoption of SOA.

Brown et al. (2006) mention the impact of SOA governance in SOA life cycle defined in four stages: modeling, assembly, deployment and control. The authors argue that SOA governance life cycle is distinct of the life cycle of services being governed. This SOA governance life cycle is characterized as a process comprising four phases: Planning: Understanding the structure of governance and the current environment; creating a starting point for IT governance; defining the scope of the governance model; driving change; Definition: Defining and refining governance processes, quality and decision making processes; defining organizational changes; defining IT changes in deployment of SOA processes; Permission: implementing the transition plan; initiating organizational changes; implementing SOA infrastructure; Measurement: measuring the effectiveness of governance process; measuring the effectiveness of organizational changes; reviewing and refining development and operational environments.

Regarding the related works, this work presents the processes at a higher level of detail. It makes explicit roles and responsibilities. Activities are presented in business process models which facilitates the execution by the participants of the SOA initiative.

\section{Process for SOA Governance}

This work proposes SOA governance processes that extend the works from Botto (2004), Spewak and Hill (1992), Kajko-Mattsson et al. (2007), Niemann (2010) and Schepers et al. (2008). The processes were detailed in macro-processes diagrams (VAC - Added Value Chain), event-oriented process flows (EPC - Event-Driven Process Chains) and function trees (Scheer, 2000). VAC corresponds to abstract descriptions (macro-process) of organization's functions that influence directly the added value of organization business (Aris, 2006). EPCs show a dynamic view, detailing the process flows and how they are supported by the business infrastructure (Davis and Brabander, 2007). Thus, the EPCs were used in processes whose activities have defined sequences. Function trees show a static view of functions, illustrating how a function is detailed in sub-functions without regard to the process flows (Davis and Brabander, 2007). Therefore, we use function trees for activities whose execution sequence is not known or varies in each organization.

The SOA governance processes and the organizational roles for them are presented as follows. A previous work presents the first insights towards SOA governance processes (Azevedo et al., 2010a). Due to space constraints some details are omitted. The processes complete specification is presented by Azevedo et al. (2010b).

\subsection{Organization Roles for SOA Governance}

We defined organizational roles which are responsible for executing the SOA governance processes (Figure 1). We propose a SOA Controller Organization Unit, which is responsible for coordinating all the SOA initiative. It includes the following roles: SOA Applications Analyst: Its responsibilities go beyond SOA-based applications that are in direct contact with the customer. Its tasks goes in the direction to ensure all customers' needs are fulfilled by the 
service, e.g., understand customer requirements, define service interfaces, define integration with existing applications, and so on; SOA Analyst: Its responsibilities includes modeling and design business processes and mapping business needs to existing or new services; SOA Architect: Its responsibility is to ensure that the infrastructure meets the needs of business and defined techniques; SOA Developer: Its responsibility is to develop and publish the services; SOA Manager: Its responsibility is to maintain and govern the SOA-based systems, according to a broader SOA strategy, ensuring that business needs are met on a strategic, tactical and operational level.

\subsection{Manage Service-Oriented Architecture}

The macro-process "Manage Service-Oriented Architecture" (Erro! Fonte de referência não encontrada.) is responsible to manage SOA approach in the organization and it is subdivided into sub-processes for building the current and future environment for SOA support, maintenance of support environment, definition of policies and standards, prospection of needed technologies and monitoring and measuring performed activities. These processes are detailed as follows.

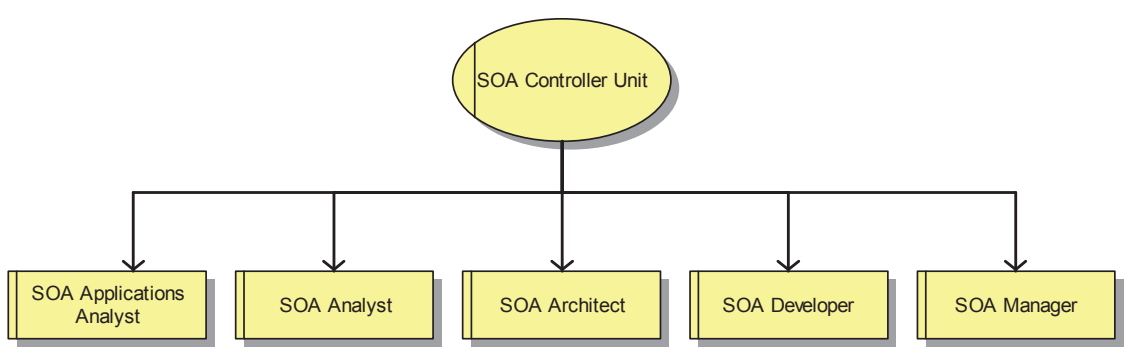

Figure 1. Organizational roles for SOA governance

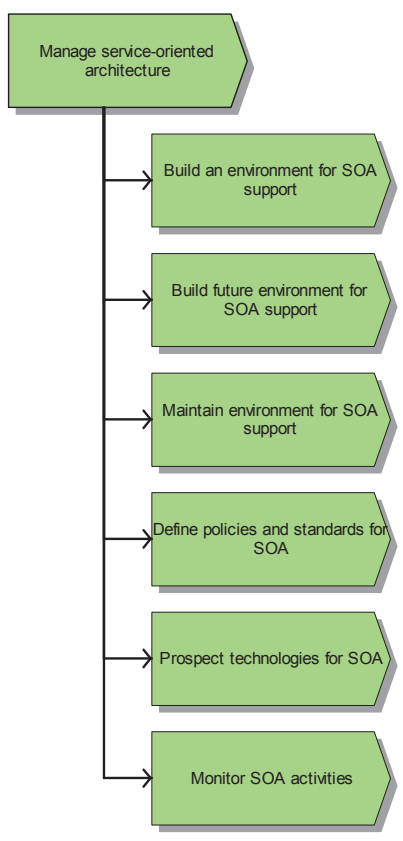

Figure 2. Process "Manage ServiceOriented Architecture"

\subsubsection{Build an Environment for SOA Support}

The process Build an Environment for SOA Support documents the current organizational environment considering existing services and systems that consume them as well as databases that are accessed by them. Besides the infrastructure used by services is documented. The activities comprised by this process are: Survey the standards currently used: Identify standards currently used for service development, e.g. standards for service implementation, service orchestration, service portfolio etc.; Survey existing services: Identify and document existing services. During this elicitation process it is important to check redundancy; Survey existing 
infrastructure: Identify the existing infrastructure, such as service bus, service registry, tools used for modeling, implementation and orchestration of services, servers for backup and provision of services etc.; Map services with the existing infrastructure: Map services to related infrastructure used to provision, monitoring, backup, clustering etc.; Map existing services and applications: Map which applications consume which services, reporting problems, difficulties and facilities in the consumption of services; Map services and databases: Map which databases are accessed by services and CRUD (Create, Retrieve, Update and Delete) operations performed by the service.

\subsubsection{Build Future Environment for SOA Support}

The process "Build future environment for SOA support" is presented in the EPC process of Figure 3. It defines the strategy and scope of the SOA initiative, including the definition of the responsibilities of stakeholders, the creation of processes to maintain the initiative and to provide the environment infrastructure. The activities that comprise it are presented as follows: Define SOA strategy: Define the strategy to implement SOA initiative, setting goals, indicators, the steps to obtain funding and human resources to the initiative; Define scope: Define the scope of the initiative, including identification of most important business processes, products that will be maintained etc.; Establish project groups: Define involved working groups; Assign responsibilities to the project groups: Define which tasks each working group must execute and what are the relationships among working groups; Define control unit: Define the organizational unit responsible for monitoring the implementation and maintaining the initiative; Assign roles to stakeholders: Define responsibilities of each stakeholder of the initiative; Define SOA processes: Define the processes of the initiative, taking into account development and management of services, registry of services in the portfolio etc.; Define infrastructures: Defining the infrastructure to be used by services (e.g., structure of services portfolio, modeling and implementation tools, technology and development standards etc.); Deploy infrastructure: Put into practice the defined infrastructure (acquisition, installation and testing of tools, and creation of services responsible to infrastructure tasks); Perform training: Conduct staff training on aspects of the SOA initiative in the organization related to their responsibilities.

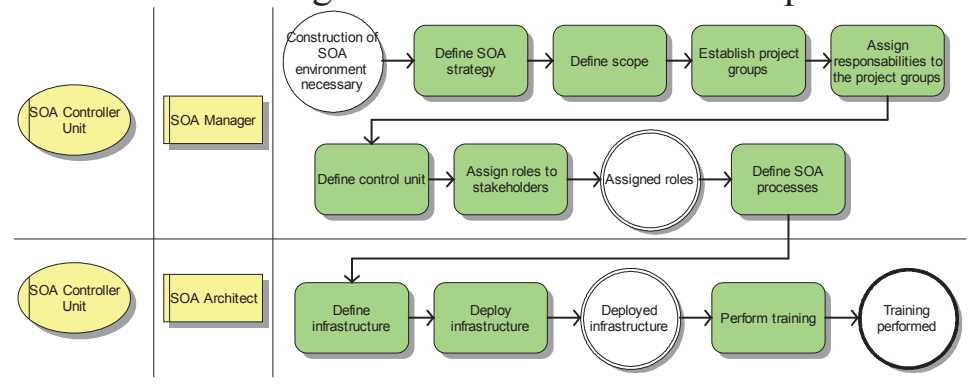

Figure 3. Build future environment for SOA support

\subsubsection{Maintain Environment for SOA Support}

The process "Maintain Environment for SOA Support" is responsible to develop and maintain available services according to business requirements and changes related to errors found, new requirements or business rules as well as changes on existing requirements or business rules. Furthermore, the infrastructure used in SOA environment is also maintained. The process is divided into other sub-processes that, due to space constraints, will not be detailed. These sub- 
processes are responsible for the following tasks.

- Maintain SOA planning: Maintain SOA planning regarding changes required in the strategy, scope, project groups and control unit and their responsibilities;

- Maintain infrastructure: Maintain the defined infrastructure for SOA environment, including updating tools, creation of user access profile and resolution of problems in the computing environment;

- Maintain services portfolio: Evaluate the need for changes in portfolio structure, ways to service discovery and in service documentations, and evaluation of the service level agreements established between consumers and service providers;

- Build services: Build new services and maintain existing services using a service development life cycle (as proposed by Gu and Lago (2007)). It includes activities such as identify services (e.g., using the method proposed by Azevedo et al. (2009) or the method proposed by Leopold and Mendling (2012) which identify services from business process models), analysis services (e.g., using the method proposed by Azevedo et al. (2011a) that takes as input the services identified from business processes models, and using information from these models to execute a set of heuristic for service analysis), design and implement services (e.g., using the approach proposed by Diirr et al. (2012) which presents the steps to be conducted to design and implement services using UML diagrams and Java technology), test services (e.g., using proposals described by Canfora and Di Penta (2009) which presents a report of results obtained in services test area, in addition of approaches of unit test, integration test, non-functional test and regression test), publish services (e.g., using the approach proposed by Arnold et al. (2007) to gather models and tools, models-based standards using formal methods that represent deployment topologies), provide services (e.g., using the SPML protocol (Oasis, 2006) proposed by OASIS and on which different data models can be used to define the actual provisioning data), monitor services (e.g., using a module for service monitoring at the Enterprise Service Bus (ESB) proposed by Bluenke and Warda (2008) - ESB is the core technology in an SOA (Hewitt, 2009) - or using the extensible monitoring model from the perspective of others proposed by Qi et al. (2010) and retire services no longer in use (as characterized by Josuttis, 2007);

- Consume services: This process corresponds to the steps performed by consumers to invoke services, such as, discovery service in a repository. If there is no service that fulfills consumer requirement, then the consumer request service development. On the other hand, if there is a service that can execute the requirement considering some adjustment, the consumer request for service maintenance. If a service composition is required, the consumer orchestrates services. The consumer also has to negotiate service contract, invoke service, test application and monitor service execution.

\subsubsection{Define policies and standards for SOA}

The process "Define policies and standards for SOA" sets policies and standards for SOA environment, including its creation, maintenance, disclosure and audit. The process is divided into other sub-processes that, due to space constraints are not detailed. These sub-processes are responsible to: Create policies and standards for SOA: This process analyze characteristics to be 
standardized, set and validate policies and standards; Maintain policies and standards for SOA: When there are opportunities for improvement (selection of standards to be analyzed, identifying opportunities for improvement and change in standards); Divulge policies and standards for SOA: Provide and advertise standard, train resources to use the standards; Control policies and standards of SOA: Define standards to be audit, collect a sample of projects, verify the use of standards, publish update rate, program audit disclosure.

\subsubsection{Prospect Technologies for SOA}

The process "Prospect technologies for SOA" presented in Figure 4 continuously prospects technologies for SOA and the activities that comprise it are described as follows: Perform search for information about tools: Search for information about tools for the SOA environment in forums, conferences, on the Web and contacting tool vendors; Assess tools: Evaluate tools executing the following steps: define evaluation criteria, compare candidate tools and select tools. Azevedo et al. (2011b) present details in how to execute tool evaluation; Define guidelines for integration technologies: Set guidelines for the integration of technology in current environment; Publish results of tools assessment: Publish the results of conducted evaluations to participants in the initiative; Assess technology viability for SOA environment: Evaluate the feasibility of implementing the selected technology; Deploy technology: Deploy the selected technologies in the environment.

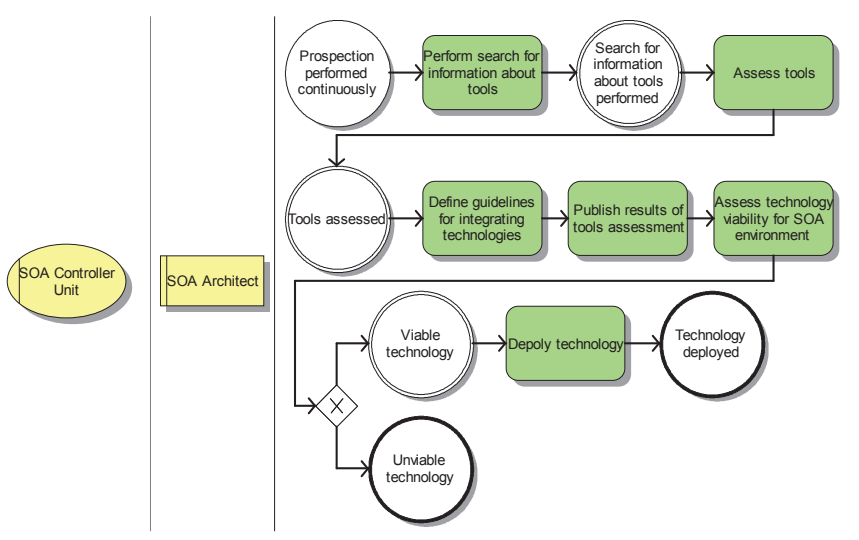

Figure 4. Prospect technologies for SOA

\subsubsection{Monitor SOA Activities}

The "Monitor SOA activities" process performs measurements and evaluations to monitor the activities executed during the SOA initiative. The activities that comprise this process are described as follows: Establish indicators: Establish quality indicators for activities related to SOA; Monitor indicators execution: Monitor the execution of initiative to compute indicators that verify whether the internal activities of the area are being carried out properly; Measure indicators execution: pointing out which gaps between planned and performed activities; Asses activities execution: Evaluate the execution of activities related to SOA, checking that they are within expected to meet the needs of the organization; Communicate achievements obtained by area: Present results achieved by the SOA initiative.

\section{Processes evaluation}


In order to evaluate the proposed processes for SOA governance the Delphi technique was used. This technique is a systematic and iterative estimation based on the experience of independent experts. These experts must be carefully selected to answer a questionnaire based on their experience. According to Rowe (2001), "Expert opinion is often necessary in forecasting tasks because of the lack of appropriate available information or using statistical procedures." In the case of this research, we used estimation because, since the processes are not yet implemented in a real environment, its applicability and reliability can only be inferred based on the experience of such professionals. A detailed view of the Delphi technique can be obtained from Rowe (1999) and Green et al. (2007). We selected five professionals with proven expertise in SOA to participate in the research. The objective of the research was evaluate whether the processes are applicable in a real environment by checking some aspects, such as, ease to understand the processes, compliance of the processes in relation to current practices, usefulness of the processes, degree of difficulty to deploy the processes, favorability to the adoption of processes, and strengths and weakness observed in the processes. During the interview, we first presented the proposed governance processes to make easy for the participants to understand the proposed processes before answering the questionnaire. The professionals have performed different roles in SOA for at least one year. They have worked as managers (2 respondents), architects (3 respondents), analysts ( 3 respondents), developers ( 1 respondent) and researchers ( 1 respondent) in SOA. All of them have been working for organizations with more than five thousand employees and whose units responsible for SOA are implemented or under implementation.

Participants pointed out some difficulties observed during the deployment of SOA in the organizations they work, which are directly related to the activities of SOA governance. The difficulties are: obtain executive support, change team culture of development and train staff, management teams (e.g., define the responsibility of each team and each person in the initiative), define the technologies to be used, define security policies, manage data exchanged between services, manage services repository, define funding model (e.g., share costs of services between different projects that use them). Moreover, participants consider the following components as important for SOA governance: management support for implementation of the initiative; definition of tools to be used; management of data exchanged by services; adherence to standards; management of repositories; and, use of indicators and metrics to monitor activities.

Currently, participants are carrying out the following activities in SOA initiative (some already implemented or under implementation) they take part: modeling, design, development and publication of services, definition of services governance processes, definition of governance standards, and control the development, quality and publication of services.

Some quality aspects of the proposed processes were asked to participants. Regarding the ease of understanding, the following question was done: "The proposed processes are easy to understand?" and, for each process, the possible answers to this question were "very easy", "easy", "medium", "difficult" and "very difficult. For all processes, 5 participants considered their understanding as easy. Participants emphasized that some aspects that ease understanding are simplicity of process design and the fact process are presented in a high level of details designed in business processes models. These observations confirm what was presented in the related work section. Besides, they mentioned that their experience in SOA as another factor that contributes to that.

Then, participants were asked about the compliance of the processes in relation to current practices of SOA initiatives in the organizations where they work. The question asked to them 
was: "Are the proposed processes in accordance with the processes used by the organization you work within a SOA initiative?" and, to answer it, participants had to classify each process in "nonconforming", "little conforming" and "conforming". Some non-conformity and little conformity responses were presented and the reasons given by participants for these results were:

- The process "Prospect technologies for SOA" in many cases is not specific to SOA, but rather a standard process established in the organization to prospect any technology. Furthermore, there are cases in which the prospection of technologies for SOA did not occur. It happens, for example, when the organization has already previous contract to acquire software from a specific vendor.;

- Unlike what is proposed in the process "Build future environment for SOA support", SOA initiatives have emerged in the organization on an ad-hoc manner and a general plan of the important aspects of its implementation was not carried out;

- In relation to the processes "Define policies and standards for SOA" and "Monitor SOA activities," the respondents indicated that few (or no) activities are performed for monitoring activities and define policies within the organizations they work. As participants indicated, without the implementation of governance processes, activities can occur without planning and monitoring, resulting in disorganization and causing problems in the implementation and maintenance of the SOA initiative.

Regarding the usefulness of the proposed processes, it was asked then to participants to "Classify the proposed processes according to the degree of usefulness to the organization where you work. Consider the following scale: Useless (1) (2) (3) (4) (5) Useful". Thus, for each process, the participants attributed a degree of usefulness. 5 participants classified the processes 'Build an environment for SOA support', 'Build future environment for SOA support', 'Maintain environment for SOA support', 'Define policies and standards for SOA' and 'Prospect technologies SOA' as processes with usefulness level equals to 4 or 5 . Respondents justified that these processes are very important for building and maintaining a successful SOA initiative. Moreover, the processes 'Build an environment for SOA' and 'Maintain environment for SOA support' were the processes which received more responses equals to 5 . These processes are considered essential to implement SOA in organizations. On the other hand, the process 'Monitor SOA activities' received only one response equal to 5, and the corresponding respondent argues that all processes are necessary to prevent future problems in the SOA initiative. The other respondents reported that, in organizations where they work, quality indicators are not defined and monitored, as there is more pressure to service provisioning than to verify the results obtained from them.

Considering the degree of difficulty to deploy the proposed processes, the following request was made to the participants: "Please indicate how you classify the difficulty to deploy each of the proposed processes in an organization." The possible answers to this question were "very easy", "easy", "medium", "difficult" and "very difficult". The participants believe that, in general, the levels of difficulty are medium and difficult. Some reasons for these classifications were: the difficulty to deploy the processes in alignment with business needs; the number of people involved in the processes that need to be adherents to them can be large (mainly in large organizations); the lack of preparation and experience of the team in SOA initiatives; and the adaptation of processes' activities according to organization specific characteristics. Thus, according to participants, the complexity involved in implementing and maintaining the SOA 
initiative is the factor that complicates the use of processes.

Then, participants were asked about the adoption of proposed processes with the following question: "Would you adopt the proposed governance processes to support a SOA initiative in the organization you work?". Despite the difficulties considered for deployment, the five participants answer "Yes". They justified that they would use all processes, as they recognize the importance they have in an SOA initiative and the benefits they can bring.

Finally, participants were asked to indicate strengths and weaknesses observed in the proposed processes. The weaknesses mentioned were: lack of activities about service quality, data quality and definition of SLAs. The strong points were: the processes are presented in a simple, objective, explanatory and well structured form (preparation, establishment of policies and standards, construction, maintenance, preparation for the future and measurement); processes are grounded in a pre-defined set of roles; processes emphasize the importance of planning and monitoring the SOA initiative rather than a disorganized implementation; the implementation of processes resulted in greater maturity of the initiative and improve the quality of services. As mentioned in the related work section and pointed out by the participants, an important characteristic of our proposal is to define the roles that are part of the initiative.

\section{Conclusion}

This paper proposed a set of processes for SOA governance based on the works of Botto (2004), Spewak and Hill (1992), Kajko-Mattsson et al. (2007), Niemann (2010) and Schepers et al. (2008). The proposed processes were, namely: Build an environment for SOA support; Build future environment for SOA support; Maintain environment for SOA support; Define policies and standards for SOA; Prospect technologies for SOA; Monitor SOA activities. In addition, the roles responsible for executing these processes were proposed: SOA applications analyst, SOA analyst, SOA architect, SOA developer and SOA manager.

In order to evaluate the processes, five participants of SOA initiatives in different organizations responded to a questionnaire about the quality and usefulness of the processes. The results indicate ease of understanding of processes and their usefulness to organizations. Some indications of little compliance or noncompliance of the proposed processes according to what is executed by participant's organizations were obtained. They emphasized that this occurs because current activities organizations perform are executed without the use of well-defined processes, as well as with little or no planning and monitoring activities. They pointed to the difficulty of implementing the proposed processes due to the complexity of a service-oriented architecture, which must be aligned to business and requires well-prepared teams. Despite the difficulties of implementation, the five participants emphasized that they would implement all processes due to its importance in an SOA initiative and the benefits that can be obtained from their implementation. They also pointed out additional strengths related to: the way in which processes are presented; definition of roles who execute the activities; better maturity and quality of SOA initiative arising from the implementation of processes; emphasis on planning and monitoring of the SOA initiative rather than an ad-hoc deployment. However, despite the opinions of the participants, there are some weaknesses in the study. The processes are not yet implemented in a real environment and therefore we cannot prove its applicability. Furthermore, the evaluation by specialists requires reading the proposed processes, which makes the evaluation time-consuming and may introduce bias if the participants do not understand the processes correctly. 
As future work, we propose the improvement of the highlighted weaknesses through addressing services and data quality more specifically, the definition of SLAs and handling deactivation of services. We also suggest using the proposed processes in real scenarios in medium and large organizations in order to assess the proposal in practice.

\section{References}

Arnold, W., Eilam, T., Kalantar, M., Konstantinou, A., Totok, A. (2007) "Pattern Based SOA Deployment". In Proceedings of the 5th international conference on Service-Oriented Computing (ICSOC), volume 4749/2007, 1-12, DOI: 10.1007/978-3-540-74974-5_1.

ARIS. (2006) "Help Documentation”. ARIS Business Architect 7.0 v. 7.0.2.234414, IDS Scheer.

Azevedo, L., Baião, F., Santoro, F., Souza, J. F. (2011a) "A Business Aware Service Identification and Analysis Approach". In IADIS International Conference Information Systems, 11-13, Avila, Spain, March.

Azevedo, L. G., Cappelli, C. Baião, F., Puntar, S. 2011b. "A Business Process Model for Software Tool Evaluation". In IADIS International Conference in Applied Computing, Rio de Janeiro, Brazil.

Azevedo, L. G.; Santoro, F.; Baião, F. (2010a) "Processos para Governança SOA baseado em Arquitetura de Tecnologia de Informação". In: Conferência IADIS Ibero-Americana WWW/Internet 2010, 2010, Carvoeiro, Algarve, Portugal.

Azevedo, L., Pereira, V., Santoro, F., Baião, F. (2010b) "Processos para Governança SOA no ponto de vista da Arquitetura de Tecnologia de Informação". Technical Reports of Department of Applied Informatics/UNIRIO (RelaTe-DIA), RT-0014/2010. Available at http://seer.unirio.br/index.php/monografiasppgi.

Azevedo, L., Santoro, F., Baião, F., Souza, J., Revoredo, K., Pereira, V., Herlain, I. (2009) "A Method for Service Identification from Business Process Models in a SOA Approach". In: 10th International Workshop on Business Process Modeling, Development and Support (BPMDS), volume 29, pp. 99-112.

Bluemke, I., Warda, M. (2008) "Monitoring services on Enterprise Service Bus". In IADIS International Journal on Computer Science and Information Systems. Vol. 3, No. 1, pp. 1-13.

Botto, R. (2004) “Arquitetura corporativa de Tecnologia da Informação”. Brasport, 268p.

Brown, W. A., Moore, G., Tegan, W. (2006) "SOA governance - IBM's approach, Effective governance through the IBM SOA Governance Management Method approach". White paper. Available at <ftp://ftp.software.ibm.com/software/soa/pdf/SOA_Gov_Process_Overview.pdf $>$.

Canfora, G.; Di Penta, M. (2009) "Service Oriented Architectures Testing: A Survey". In Software Engineering, Springer-Verlag, Berlin, Heidelberg.

Davis, R.; Brabander, E. (2007) “ARIS Design Platform: Getting Started with BPM”. Springer.

Deler, P., Weinreich, R. (2006) "Models and Tools for SOA Governance". In International Conference on Trends in Enterprise Application Architecture, Berlin, Germany, Nov. 29-Dez. 1, Springer Lecture Notes on Computer Science (LNCS), Volume 4473/2007, pp. 112-126. Diirr, T., Azevedo, L. G., Faria, F., Santoro, F., Baião, F. (2012) "Practical Approach for Service Design and Implementation". In IADIS International Conference Information 
Systems 2012, pp. 197-204, 10-12 March, Berlim, Alemanha.

Erl, T. (2005) "Service-Oriented Architecture: Concepts, Technology, and Design". PrenticeHall, Englewood Cliffs, August, 792 p.

Green, Armstrong, Graefe. (2007) "Methods to Elicit Forecasts from Groups: Delphi and Prediction Markets Compared". Forthcoming in Foresight: The International Journal of Applied Forecasting, Fall.

Gu, Q., Lago, P. (2007) “A stakeholder-driven Service Life Cycle Model for SOA”. In 2nd international workshop on Service oriented software engineering, pp. 1-7.

Hewitt, E. (2009) “Java SOA Cookbook”, O’Reilly, April, 740 p..

Josuttis, N. (2007) “SOA in Pratice: The Art of Distributed System Design”. O'Reilly.

Kajko-Mattsson, M., Lewis, G. A., Smith, D. B. (2007) "A Framework for Roles for Development, Evolution and Maintenance of SOA-Based Systems". In Proceedings of the International Workshop on Systems Development in SOA Environments, Washington, USA.

Leopold, H., Mendling, J. (2012) "Automatic Derivation of Service Candidates from Business Process Model Repositories". In: 15th International Conference on Business Information Systems (BIS 2012), May 21-23, Vilnius, Lithuania.

Marks, E. A. (2008) "Service-Oriented Architecture Governance for the Services Driven Enterprise", John Willey \& Sons Inc, 330 p.

Niemann, M., Miede, A., Johannsen, W., Repp, N., Steinmetz, R. (2010) "Structuring SOA governance". In International Journal on IT/Business Alignment and Governance, volume 1, issue 1,18 pages., $58-75$.

Oasis. (2006) "OASIS Service Provisioning Markup Language (SPML) v2”. Available at $<\mathrm{http}$ //www.oasis-open.org/committees/tc_home.php?wg_abbrev=provision>

Qi, K., Han, Y., Zhao, Z., Fang, J. (2010) “An Adaptive Service Monitor Providing Runtime Extensibility". In Proceedings of the 2010 Fifth IEEE International Symposium on Service Oriented System Engineering IEEE Computer Society, Washington, USA.

Jeanne Ross. (2011) "Enterprise Architecture Comes of Age". ICIS 2011 Proceedings. Paper 1.

Rowe, G., Wright, G. (1999) "The Delphi technique as a forecasting tool: issues and analysis". International Journal of Forecasting, Volume 15, Issue 4, October.

Rowe, G., Wright, G. (2001) "Expert opinions in forecasting: The role of the Delphi technique." In Armstrong, J. S. (Ed.): Principles of forecasting: a handbook for researchers and practitioners, Kluwer Academic Publishers, 125-144, Norwell, MA.

Schepers, T. G. J., Iacob, M. E., Van Eck, P. A. T. (2008) "A lifecycle approach to SOA governance". In Proce. of the 2008 ACM symposium on Applied computing, pp. 1055-1061.

Scheer, A. W. (2000) “ARIS - Business Process Modelling”. Springer, 238 p.

Spewak, S. H., Hill, S. C. (1992) "Enterprise Architecture Planning: Developing a Blueprint for Data, Applications and Technology", John Wiley \& Sons Inc, 393 p., October. 